\title{
La Torre de Comares en peligro de ruina. Afecciones de la estructura más significativa de la Alhambra en los siglos XVI y XVII
}

The Tower of Comares in danger of collapse. Affections in the most significant structure of the Alhambra during the sixteenth and seventeenth centuries

\section{Virginie Claude Brazille Naulet ${ }^{a}$, Antonio Orihuela Uzal ${ }^{\text {b }}$, Luis José García-Pulido ${ }^{\text {c }}$}

\author{
${ }^{a}$ Asesora externa del Patronato de la Alhambra y del Generalife, Granada, Spain, virginiabraz@yahoo.fr

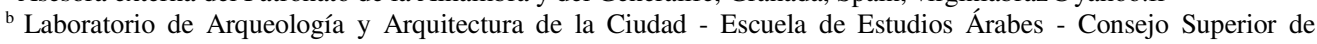 \\ Investigaciones Científicas, Granada, Spain, orihuela@eea.csic.es \\ c Laboratorio de Arqueología y Arquitectura de la Ciudad - Escuela de Estudios Árabes - Consejo Superior de \\ Investigaciones Científicas, Granada / Departamento de Arte y Arquitectura - Universidad de Málaga, Málaga, Spain, \\ luis.garcia@uma.es
}

\begin{abstract}
This article develops a research on the outside layout of the Tower of Comares in the Alhambra (Granada), studying its transformations. It is focused on the interventions that took place at the most critical moment in its history. Between the last decades of the sixteenth century and the first ones of the seventeenth century, its general state of abandonment, the explosion of a nearby powder mill and the bad restauration practices applied in some cases, brought it to its structural limits, threatening its ruin. This process could fortunately be reversed after several technical reports on its injuries and actual conservation estate. If neglected, it would have led to the collapse of this paradigmatic example of the Nasrid regal defensive architecture.
\end{abstract}

Keywords: Alhambra, north walls, Tower of Comares, Hall of the Throne, heritage conservation.

\section{Introducción}

La construcción de la Torre de Comares durante el reinado de Yūsuf I (1318-1354) supuso la ampliación hipertrofiada de una torre previa de menor tamaño del siglo XIII existente en el lienzo norte del recinto de la Alhambra, y constituyó el rasgo más visible de la transformación de un frente murario con un carácter eminentemente defensivo a la proyección de la imagen del poder (Fig. 1) sobre la ciudad de Granada y su territorio (Orihuela Uzal, 1996, pp. 81-102).

En la zona del Patio de la Reja, el antiguo camino de circunvalación existente hasta las reformas del siglo XIV, pasaba paralelo a la gale- ría sur del Patio de Lindaraja (Vílchez Vílchez, 1985-1986, pp. 360), tal y como viene representado en la Planta Baja de la Casa Real de la Alhambra de 1927. En ella, Leopoldo Torres Balbás indicó con línea intermitente la continuidad hacia el este del camino de circunvalación, tras una exploración realizada en 1925 (APAG/ Colección de Planos/ P-001026).

El camino de circunvalación y el paso de ronda del nivel del adarve circundaban toda la Alhambra, y en los lugares en donde no aparecen han sido cercenados o tapados debido a las distintas transformaciones realizadas a lo largo de los 
siglos (Bermúdez Pareja, 1973, pp. 55-56). Esto pudo ser comprobado en una excavación realizada en 1981, proponiéndose la existencia de dicho camino y dos pasos de ronda sobre una anterior muralla y antemuralla que habrían dejado su impronta en los espacios vacíos existentes en los sótanos de la mitad meridional de la torre, tales como la Sala de las Ninfas, o las galerías y las dos salas de levante del Patio de la Reja y las naves abovedadas de poniente (Vílchez Vílchez, 1985-1986, pp. 355, 360; Orihuela Uzal, 1996, pp. 99-100). La existencia de estos espacios vacíos (Fig. 2), rellenos o no en el momento de ampliación de la torre, acabarían generando problemas de estabilidad en la estructura de la torre que aún quedan por resolver en nuestros días.
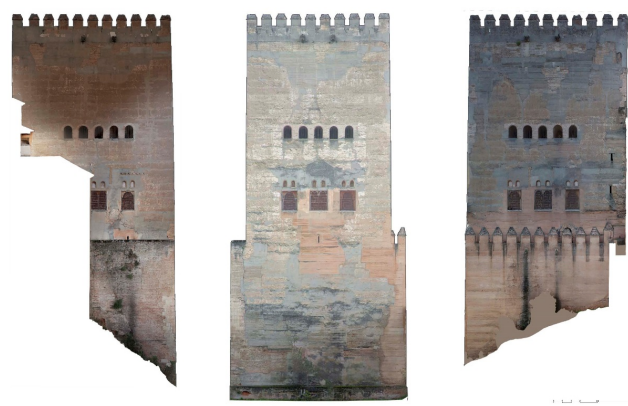

Fig. 1. Ortofotos de los alzados este, norte y oeste de la Torre de Comares (Autores, 2018).

La decadencia de estas construcciones en el último periodo nazarí pudo haber tenido un punto de inflexión en 1431. El 27 de junio, pocos días después de la entrada de Juan II en la Vega de Granada y que se produjese la batalla de la Higueruela, un primer terremoto causó la caída de muros y torres (Torres Balbás, 1951, p. 187). Las series de temblores que siguieron a esta primera sacudida propició que se cayeron trozos de las murallas de la Alhambra (Casares López, 2008, p. 239).

En las últimas décadas de la dinastía nazarí, la precariedad en la que se encontraba el reino y el pago periódico de impuestos a Castilla para evitar el conflicto armado, no debió de propiciar recursos sobrados ni ocasiones oportunas para reparar edificaciones de tan extrema fragilidad, necesitadas de continua atención, de tal modo que muchas de las construcciones de la colina roja pasaran ruinosas al poder de los Reyes Católicos en 1492.

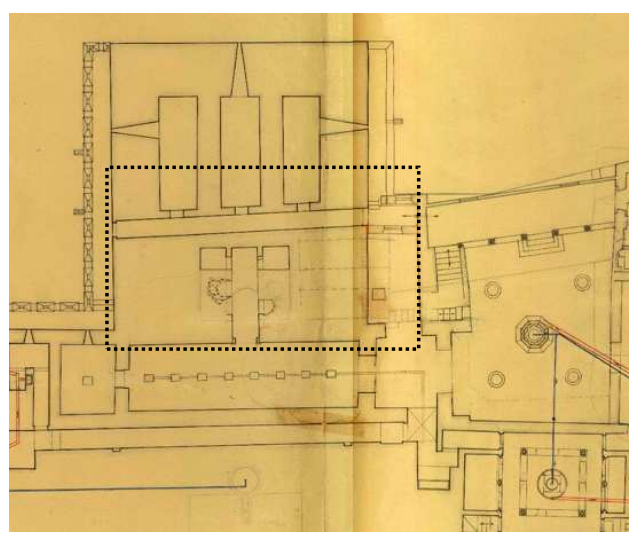

Fig. 2. Detalle de los sótanos de la parte meridional de la Torre de Comares en el plano titulado "Planta parcial con conducciones de la Torre de Machuca y los Palacios Nazaríes" (Prieto-Moreno y Pardo, Francisco, ca. 1940, APAG/ Colección de Planos/ P-000018).

Un francés asistente a la capitulación, cuyo relato está fechado el 10 de enero de 1492 en Granada, alude a la partida inminente de Fernando e Isabel a Aragón, después de haber hecho reparar algunas torres y corregir el mal estado de otras. Ese mismo año comenzaron las obras de reparación del alcázar, según consta en la correspondencia mantenida entre los Reyes Católicos y su secretario Hernando de Zafra entre 1492 y 1494, que manifiesta la realización de importantes obras en la Alhambra, algunas de ellas de fortificación (Torres Balbás, 1951, pp. 187-188, 193 y 196). Este hecho fue constatado por Jerónimo Münzer, quien, a su paso por Granada en 1494, observó que muchos mudéjares "[...] trabajan en las obras de reparación de la Alhambra o de otras reales posesiones" (Münzer, 1494, p. 89).

\section{La Torre de Comares en el siglo XVI}

Durante los primeros años del siglo XVI se transformaron las zonas palaciegas entre Comares y Leones, y en 1546, bajo la dirección de Alonso de Montalvo, se produjo una intensa intervención en la Torre de Comares, añadiéndole dos estribos por el norte a su base. En 1552, se colocaron tirantas entre ella y la Sala de la Bar- 
ca, para asegurarla (Vilar Sánchez, 2016, p. 209;

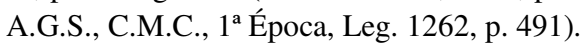

A partir de 1547 y en 1553, se realizaron restauraciones en las murallas, torres y en los palacios de Comares y de los Leones. También se trabajó en las torres de la fortaleza, como aparece en un asiento del día 2 de diciembre de 1553 , en el que se pagaron a Baltasar de la Cruz 3200 maravedíes por aparejar y solar 4000 ladrillos en las torres de la Alhambra (Casares López, 2008, pp. 248 y 253 ).

Tras la rebelión de los moriscos (1568-1571), cayeron considerablemente los ingresos destinados a las obras (Galera Mendoza, 2014, p. 26). La sublevación morisca y la consecuente expulsión del reino de Granada de esta población, despojó a la Alhambra de sus principales ingresos, tal y como quedó reflejado en los datos del libro de cuentas de Ceprián León. Así, en los años transcurridos desde 1572 a 1574, las obras continuaron con los remanentes de los materiales de sus almacenes, y después se vieron paralizadas hasta 1584 (Casares López, 2008, pp. 260262). En 1588 se acometieron obras de mantenimiento en la Torre de Comares, en concreto en la bóveda, yeserías, solerías y en las ventanas, donde se colocaron guardapolvos.

\section{Un incidente destructivo para la Alhambra en la última década del siglo XVI}

En 1590 se produjo el incendio y explosión de un molino de pólvora cerca de río Darro, en la ladera norte de la Alhambra, acontecimiento que afectó tanto al Palacio de Leones como al de Comares, que habían sido objeto de restauraciones ya casi terminadas cuando ocurrió el desastre. La deflagración hizo que saltaran por los aires cristales, puertas y ventanas. Además, el molino lanzó materiales en llamas sobre toda la Alhambra, provocando incendios en varios lugares. Los más afectados fueron todos los edificios del frente septentrional que daban al río, pero, sobre todo, la Torre de Comares, la Sala de los Abencerrajes, la Sala de los Mocárabes en el Palacio de los Leones y los cuartos nuevos. Todos los reparos que se habían estado haciendo sufrieron desperfectos (Casares López, 2008, p. 276; Bermúdez Pareja, 1966, p. 79).

\subsection{Reconocimiento de daños}

El Alcaide de la Alhambra, Miguel Ponce de León, encargó el 18 de febrero de 1590 un informe al maestro aparejador de las obras reales, Juan de la Vega, "sobre los daños debidos a la explosión producida por el incendio del taller de un polvorista situado entre la Puerta de Guadix y el río Darro, cerca de San Pedro" (Casares López, 1973, p. 54). Según la información aportada por Juan de la Vega, la voladura del molino de pólvora, provocó daños en el lateral este de la Torre de Comares y en el Salón del Trono, donde destruyó las celosías vidriadas, lo que restase de los ajimeces medievales y los demás elementos de la carpintería de los huecos y ventanas (Castilla Brazales, Orihuela Uzal, 2002, p. 353, APAG- L-6-27; Bermúdez Pareja, 1966, p. 82). También afectó a las yeserías de los muros, arcos y bóvedas (Torres Balbás, 1931, APAG-2000.038).

Como consecuencia de ello se compraron 12000 ladrillos para el reparo de la Torre de Comares (Casares López, 1987, p. 55), y a fines de ese año se libraron varias cantidades por partidas de rasillas mazaríes, azulejos y alizares (Torres Balbás, 1931, APAG-2000.038).

Durante 1592 y 1593 se hicieron reparos en el Palacio de Comares, que quedaron registrados en los pagos del carretero Juan de Montoya por el acarreo de piedra franca de las canteras de Santa Pudia (Casares López, 2008, pp. 281 y 284). En 1595 se acabó la guarnición de 15 vidrieras grandes y 18 pequeñas, rehaciéndose además 4 pedazos de yesería blanca en la cuadra principal de Comares. (Torres Balbás, 1931, APAG-2000.038).

\section{Reformas inadecuadas en la Torre de Co- mares}

Los graves daños originados por la explosión del polvorín en la estructura de este sector de las murallas y en los palacios, se vieron agravados por los originados por el abandono, la falta de fondos, los agentes meteorológicos, los terremotos y la realización de una serie de reformas inadecuadas. 
En 1624 se llevaron a cabo nuevas obras y dos años más tarde se arreglaron las ventanas de la Torre de Comares. Éstas se podían abrir por medio de una especie de rieles adosados a la fachada exterior y además tenían unos protectores o voladizos con canecillos situados por encima de ellas (Vilar Sánchez, 2016, pp. 209210).

En el primer tercio del siglo XVII se quiso habilitar los sótanos de la Torre de Comares para residencia de verano de los alcaides y gobernadores (Torres Balbás, 1931, APAG-2000.038). Para ello se comunicaron las pequeñas bóvedas de la torre primitiva con las tres grandes de la ampliación hacia el norte de la torre realizada en época de Yūsuf I, rompiendo el muro septentrional de la bóveda central antigua. En el año 1627 se practicó un ancho hueco en el testero norte de la bóveda central más reciente, con objeto de instalar un balcón y dos ventanas con rejas en los alzados este y oeste (Orihuela Uzal, 1996, p. 100).

Desde 1628, la torre y los edificios colindantes daban claras muestras de deterioro, teniendo que solicitar el maestro mayor y el veedor de las obras reales fondos a la administración real para subsanar los daños y evitar que llegasen a más (Vilar Sánchez, 2016, p. 210). No debieron de acometerse, pues en 1638 la torre amenazaba ruina (Torres Balbás, 1931, APAG-2000.038). Ese mismo año, se instalaron unos estribos en la base de la Torre de Comares (Vilar Sánchez, 2013, pp. 60-61; APAG, Legajo 152-1).

A partir de la década de 1640 se sucedieron los informes y las reparaciones, dado que el marqués de Mondéjar ordenó que los maestros de obras estudiasen las intervenciones que eran precisas.

\subsection{Informe de Miguel Guerrero y Juan López (1644)}

En abril de ese año, Miguel Guerrero maestro mayor de la catedral, junto con Juan López, maestro de albañilería y alarife de la ciudad, reconocieron la Torre de Comares, que daba evidentes señales de ruina, viendo los reparos necesarios (Casares López, 1973, p. 56; Galera Mendoza, 2014, p. 194).
Señalaron dos grietas de considerable longitud en los pilastrones centrales que daban al norte, que sembraron la inquietud entre los encargados del mantenimiento de la Alhambra. Lo mismo ocurrió con la grieta que se abrió en el suelo, ya que llegaba hasta las bóvedas de los acuartelamientos subterráneos.

La solución que dieron para este reparo fue la de macizar todas las ventanas bajas con piedra, disminuir los vanos, así como proceder al derribo de las almenas de la Torre de Comares para aliviar su peso. Asimismo, expresaron la necesidad de que "La bóveda que cubre la torre, que tiene dos ladrillos de grueso, que se derribe por el empuje que hace y porque esta falta de estribos con arreglo al hueco de la torre, pues teniendo cuarenta pies debe tener de estribos la cuarta parte, y que se sustituya con una armadura" (Torres Balbás, 1931, APAG-2000.038).

Ese mismo año, el maestro mayor de las obras reales de la Alhambra, Fernández Lechuga, dio cuenta a Felipe IV del daño y ruina que amenazaba a la Torre de Comares y los reparos que necesitaba (Vilar Sánchez, 2016, p. 211).

Según se desprende del documento, se dedicó a observar diariamente la grieta y pudo ver cómo iba abriéndose cada vez más: "[...] la dicha quiebra sale afuera y sin llegar al cimiento de la dicha torre se desgaja y va a salir al balcón de la bóveda" (Casares López, 1973, pp. 56-57).

Para su reparación propuso dos planes a seguir. El primero consistía en descargar la torre, deshaciendo la bóveda de ladrillo que la cerraba y todas las almenas y los gruesos de las paredes:

"[...] hasta que encima dellas se pueda sentar una armadura de madera, a cuatro vertientes y cubierta de teja, que quede a tal altura que no tope con la bóveda de madera, que oy está hecha [...]" (Casares López, 1973, pp. 56-57).

No existen representaciones de la Torre de Comares sin almenas, pero podemos imaginar que se llegaron a derribar, dado que las actuales son de ladrillo, y no de tapia. Su alzado actual aún refleja un error de replanteo al tener un mayor espaciamiento entre las dos primeras almenas en el alzado este (Fig. 3). 


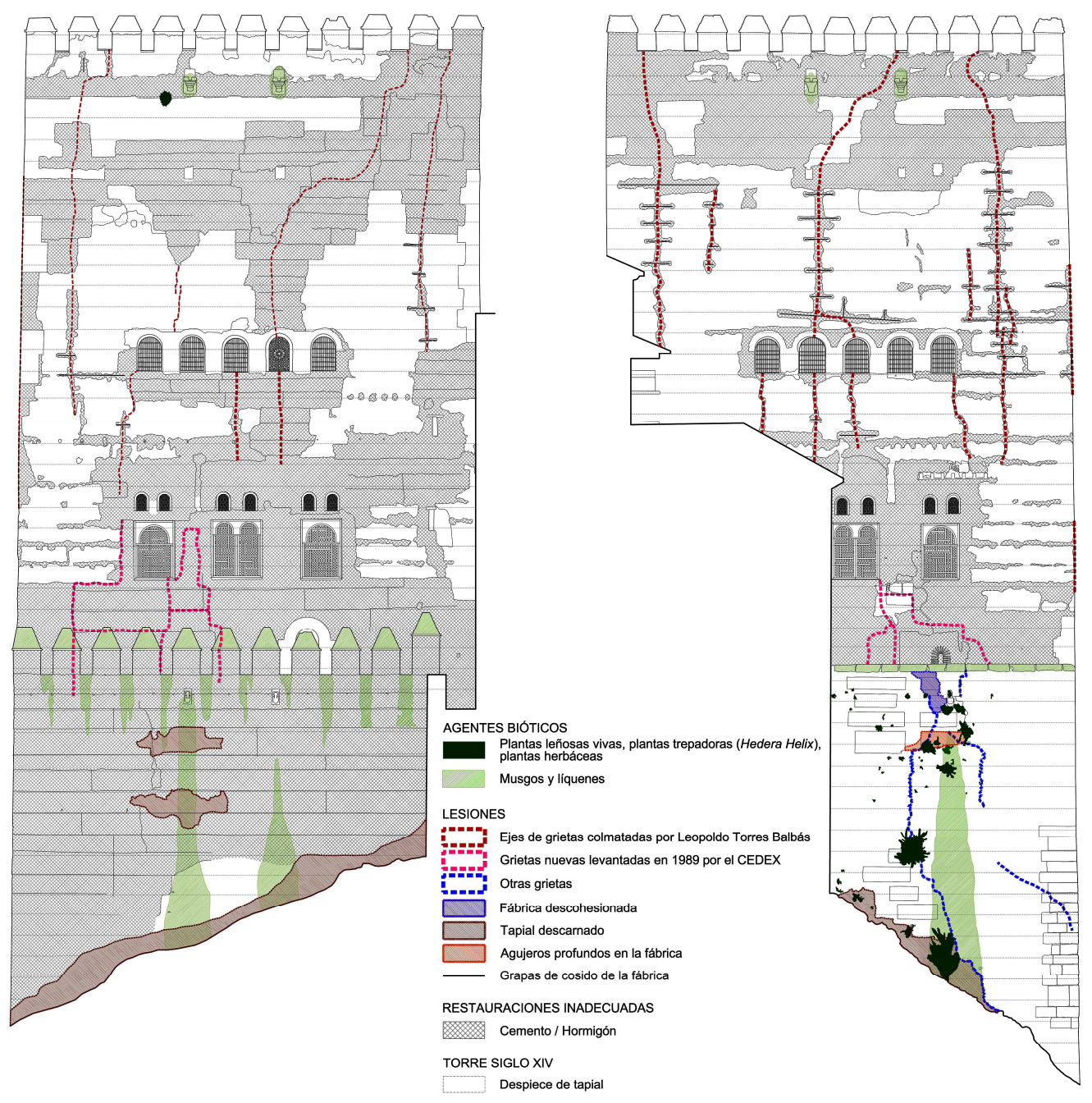

Fig 3. Alzados oeste (izquierda) y este (derecha) de la Torre de Comares, con las lesiones que presentan (Autores).

El segundo plan para repararla sería aparejarle dos estribos en las esquinas "[...] que cada uno tenga de frente cinco varas y que sean de tova con muchas piedras largas que a trechos entren a tiçon en dichos estribos y que entren una dentro en la pared de dicha torre, para que reciban y ayuden a sustentar hacia arriba, y lo mismo se a de hacer con los sillares que arriman con dicha torre [...]" (Casares López, 1973, p. 57).

\subsection{Informe de Pedro Pérez Merino (1670)}

Pedro Pérez Merino, aparejador y maestro mayor de las obras reales hasta 1684 , redactó un informe en 1670 acerca de las reparaciones necesarias, indicando lo siguiente:

"[...] dixo que ha visto y reconocido los reparos de que necesitan las dichas casas reales y entre los demás que son precisos y más forzosos es la torre de Comares en unos pilastrones que están entre la ventana y ventana de la parte de levante, norte, y poniente en los balcones, para lo cual es necesario que los dichos pilastrones se reparen haciéndolos de nuevo de piedra volviéndolos a chapar de la misma forma y manera que hoy están" (Galera Mendoza, 2014, p. 193). 


\subsection{Primeras obras de restauración (1671)}

La restauración de la Torre de Comares comenzaría bajo la maestría de Pedro Pérez Merino y se prolongaría hasta la de Juan de Rueda Alcántara, acometiéndose entonces la restauración completa (Galera Mendoza, 2014, pp. 177 y 190).

Pedro Pérez redactó las condiciones de la licitación para sacar al pregón la extracción de cien varas de piedra de Alfacar de la cantera del rey. El cantero Andrés Gómez trabajó labrando los sillares para la reparación de la Torre de Comares y entre 1673 y 1676 se llevó a cabo bajo la dirección de Pedro Pérez el calzamiento de la pared de la puerta principal de la Torre de Comares (Galera Mendoza, 2014, pp. 195 y 306).

\subsection{Informes de Juan de Rueda Alcántara y de José del Baño Montañés (1686)}

El 5 de febrero de 1686, Juan de Rueda Alcántara, maestro mayor de las obras de la ciudad de Granada y de las reales de la Alhambra, redactó un informe en el que refirió haber visto y reconocido todo el recinto, y en particular la Torre de Comares (Orihuela Uzal, 1996, p. 100), constatando el estado ruinoso de la misma y señalando las consecuencias que podían acarrear su derrumbe. Hasta el 4 de abril de ese año realizó nuevos reconocimientos de la torre y examinó los desperfectos, realizando un presupuesto del coste de las obras y un informe definitivo con diagnosis y mediciones, destinado a Carlos II (Casares López, 1973, p. 57). El 29 de mayo de 1686, entregó la planta del Salón del Trono y la sección transversal de la Torre de Comares por el centro de dicha sala y con los sótanos situados por debajo (Archivo General de Simancas, M.P.D. 13, 60 y M.P.D. 38, 105).

En su primer informe propopía:

"[...] calzar seis pilastrones de piedra de Alfacar, cantera de su majestad, macizando para ello las ventanas como lo están las que miran a levante, alegrar todas las rajas y macizarlas echando si fuere necesario algunas aspas embebidas asi por de fuera como por de dentro [...]" (Casares, 1973, 59) (Fig. 4).

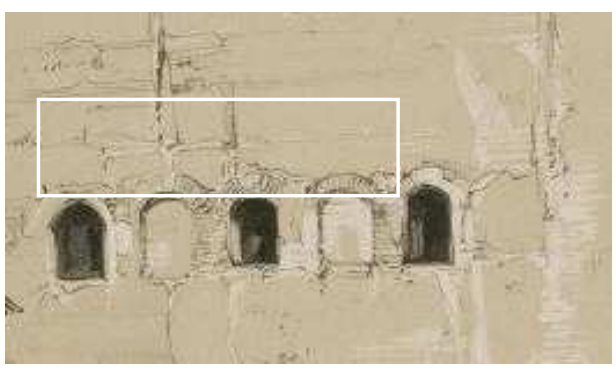

Fig. 4. Dibujo de John F. Lewis, 1833-34. Detalle.

El 21 de mayo de 1686, Juan de Rueda Alcántara, Salvador Marín, Manuel González, Diego González, Alonso Gallardo, Francisco Rodríguez y Pedro García, maestros alarifes de albañilería; Manuel Risueño, Gerónimo de Molina, Francisco Gutiérrez y Andrés Alonso, maestros alarifes de carpintería, y Francisco García, alarife, llevaron a cabo el reconocimiento de la Torre de Comares desde los cimientos hasta las almenas (Torres Balbás, 1931, APAG-2000.038). Todos estos informes coincidían en indicar que dicha estructura se estaba hundiendo por haberse removido y cuarteado sus paredes, de forma que se había empezado a romper y saltar lo labrado de ellas (Casares López, 1973, p. 55).

En 1687, se elaboró un nuevo reconocimiento dirigido por Juan de Rueda (Torres Balbás, 1931, APAG-2000.038).

Las obras se llevaron a cabo entre 1688 y 1691. Comenzó desde la parte alta hasta abajo. Lo primero fue la sustitución de la bóveda esquifada bajo la cubierta de la torre por una armadura para aliviar el peso (Fig. 5). En enero de 1689 la armadura estaba a punto de terminarse y ese mismo año se elaboró un informe de visita de obra de Juan de Rueda, dando el visto bueno el 26 de noviembre. Para subirla a lo alto de la torre, los adjudicatarios de la obra, Diego López y Diego del Arco, solicitaron licencia para colocar un torno en el bosque desde donde podría elevarse con más facilidad. Posteriormente se realizó el calzamiento de la torre, reforzando los espacios entre ventanas (Fig. 6) y los pilastrones con piedra de Alfacar. Aunque las condiciones establecían el refuerzo hasta ocho varas de altura, solo se llegó hasta unas seis varas porque la fábrica de la torre seguía siendo de un tapial hormigonado más fuerte que el refuerzo que se 
estaba haciendo, tal y como indicó Juan de Rueda (Galera Mendoza, 2014, pp. 199-204).

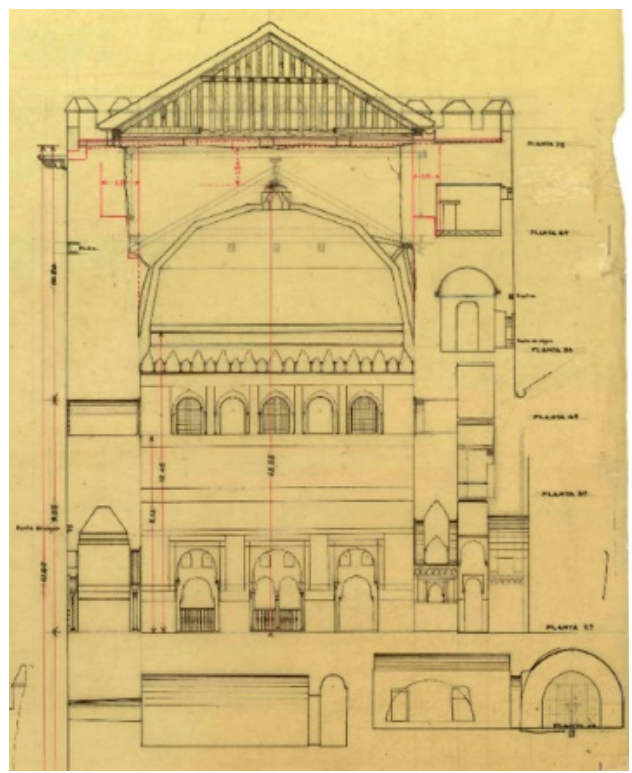

Fig. 5. Cubierta a cuatro aguas, ventanas altas macizadas. Torres Balbás (1931, APAG-2000.038) dibujó a lápiz una hipótesis de torre intermedia con un techo a cuatro aguas, sujetando la bóveda de madera a la misma altura que las gárgolas integradas en la fábrica de tapial, debajo de las actuales (APAG-P.000127).

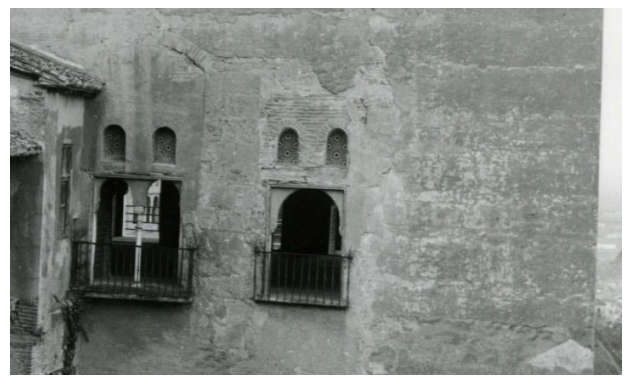

Fig. 6. Fotografía del año 1925. Los paramentos en mal estado dejan ver los recalces de piedra de Alfacar entre las ventanas y los arcos de descarga apoyados encima.

El 6 de febrero de 1691 se realizó la visita de fin de obra del alarife de carpintería de la ciudad. Reconocieron la armadura, el tejado y su solería, el aderezo de almenas y el vaciado de la bóveda, declarando que se habían cumplido con su tenor según dichas condiciones (Galera Mendoza, 2014, p. 205).

\section{Conclusiones}

En época de los Reyes Católicos se acometió una política de atención a la Alhambra, al ser considerada como un trofeo, concretándose en numerosas obras de restauración y conservación. Entre otras actuaciones, se llevó a cabo la reforma y consolidación de las fortificaciones y la adaptación de los apartamentos reales.

Durante el reinado de Carlos V, se actuó en otras habitaciones reales nuevas, destinadas a su persona y a Isabel de Portugal. Se focalizaron los fondos en la construcción del Palacio de Carlos V.

Se llevó a cabo la reforma del Peinador de la Reina y la conexión con la pasarela, las galerías del Patio de la Reja y el Proyecto del Palacio de Carlos V, que conllevó la destrucción de la Sala de las Helias y la colmatación del adarve del Patio de la Reja (Vílchez Vílchez, 1985-86, pp. 377).

Durante el reinado de Felipe II se produjo la rebelión de los moriscos (1568-1571). Tras este periodo se realizaron obras mínimas de mantenimiento en la Casa Real Vieja.

A finales del siglo XVI, la Torre de Comares se encontraba en peligro de ruina, debido, entre otros motivos, a los espacios vacíos existentes en el subsuelo de su mitad meridional (procedentes de la evolución de la muralla norte de la Alhambra y la ampliación de la torre en el siglo XIV), a la explosión del molino de pólvora en 1590 y a las reparaciones inadecuadas en las reformas efectuadas.

De las intervenciones llevadas a cabo en este periodo, se constata que la torre tenía bóveda de ladrillo que se substituyó por una techumbre a cuatro aguas. Se llevaron a cabo actuaciones de recalce de la torre. Muchas obras tuvieron trascendencia en la configuración del estado actual de los alzados.

Todas ellas proporcionan claves para la comprensión de las patologías actuales de la torre, así como la configuración del Patio de la Reja y las conexiones de pasillos y escaleras en la parte este y oeste de la torre. 


\section{Bibliography}

Bermúdez Pareja, J.; Moreno Olmedo, M.A. (1966). "Documentos de una catástrofe en la Alhambra", Cuadernos de la Alhambra, 2, pp. 77-88.

Cabrera Orti, M.A.; Vílchez Vílchez, C. (2005). "El primitivo foso de la zona del Partal de la Alhambra", Revista del Centro de Estudios Históricos de Granada y su Reino, 17, pp. 159-168.

Casares López, M. (1973). "Documentos sobre la Torre de Comares (1686)”, Cuadernos de la Alhambra, 9, pp. 5366.

Casares López, M. (2008). Las obras reales de la Alhambra en el Siglo XVI: Un estudio de los libros de cuentas de los pagadores Ceprián y Gaspar de León, Tesis doctoral, Universidad de Granada, Granada.

Castilla Brazales, J.; Orihuela Uzal, A. (2002). En busca de la Granada andalusí, ilustraciones de Miguel Sobrino González, Ed. Comares S.L., Granada.

Galera Mendoza, M.E. (2014). Arquitectos y maestros de obras en la Alhambra (siglos XVI-XVIII): artífices de cantería, albañilería, yesería y forja, Comares, Universidad de Granada, Granada.

Puyol, J. (1924). “Jerónimo Münzer. Viaje por España y Portugal en los años 1494 y 1495. (Conclusión)”, Boletín de la Real Academia de Historia, 84, pp. 197-279.

Orihuela Uzal, A. (1996). Casas y palacios nazaríes. Siglos XIII-XV, El Legado Andalusí - Lunwerg Editores S.A., Barcelona.

Torres Balbás, L. (1931). Proyecto de restauración de la Torre de Comares, Archivo del Patronato de la Alhambra y Generalife, Expediente APAG 2000/038.

Torres Balbás, L. (1947). “Ajimeces”, Al-Andalus, XII. Crónica Arqueológica de España Musulmana, XXI, pp. 1129.

Torres Balbás, L. (1949). "Ventanas de vidrios de colores en los edificios hispanomusulmanes", Al-Andalus, XIV. Crónica Arqueológica de España Musulmana, XXIV, pp. 197-201.

Torres Balbás, L. (1951). "Los Reyes Católicos en la Alhambra”. Al-Andalus, XVI. Crónica Arqueológica de la España Musulmana, XXVIII, pp. 185-205.

Vilar Sánchez, J.A. (2013). Obras en la Alhambra. Trascripción, índices y análisis del legajo 152-1 del Archivo Histórico de la Alhambra, Ed. Alhulia, Granada.

Vilar Sánchez, J.A. (2016). Murallas, torres y dependencias de la Alhambra. Una revisión de los avatares sufridos por las estructuras poliorcéticas y militares de la Alhambra, La biblioteca de la Alhambra, Ed. Comares y Patronato de la Alhambra y Generalife, Granada.

Vílchez Vílchez, C. (1985-86). "La disposición musulmana del Patio de la Reja de la Alhambra de Granada. Memoria de excavación", Cuadernos de Arte de la Universidad de Granada, 17, pp. 353-378. 